\title{
Article
}

\section{Hyers Stability and Multi-Fuzzy Banach Algebra}

\author{
Parvaneh Lo' lo $^{\prime 1}$, Ehsan Movahednia ${ }^{1}$ (D) and Manuel De la Sen ${ }^{2, *(D)}$ \\ 1 Department of Mathematics, Behbahan Khatam Alanbia University of Technology, Behbahan 6361647189, \\ Iran; lolo@bkatu.ac.ir (P.L.); movahednia@bkatu.ac.ir (E.M.) \\ 2 Institute of Research and Development of Processes, University of Basque Country, \\ Campus of Leioa (Bizkaia), 48080 Bilbao, Spain \\ * Correspondence: manuel.delasen@ehu.es
}

check for updates

Citation: Lo'lo' ${ }^{\prime}$ P.; Movahednia, E.; De la Sen, M. Hyers Stability and Multi-Fuzzy Banach Algebra. Mathematics 2022, 10, 106. https:// doi.org/10.3390/math10010106

Academic Editor: Hsien-Chung Wu

Received: 21 November 2021

Accepted: 25 December 2021

Published: 29 December 2021

Publisher's Note: MDPI stays neutral with regard to jurisdictional claims in published maps and institutional affiliations.

Copyright: (c) 2021 by the authors. Licensee MDPI, Basel, Switzerland. This article is an open access article distributed under the terms and conditions of the Creative Commons Attribution (CC BY) license (https:// creativecommons.org/licenses/by/ $4.0 /)$.

\begin{abstract}
In this paper, we define multi-fuzzy Banach algebra and then prove the stability of involution on multi-fuzzy Banach algebra by fixed point method. That is, if $f: A \rightarrow A$ is an approximately involution on multi-fuzzy Banach algebra $A$, then there exists an involution $H: A \rightarrow A$ which is near to $f$. In addition, under some conditions on $f$, the algebra $A$ has multi $C^{*}$-algebra structure with involution $H$.
\end{abstract}

Keywords: stability; multi fuzzy Banach algebra; fixed point theory

MSC: Primary 39B82; 34K36; Secondary 47B48; 47H10

\section{Introduction}

H. G. Dales and M. E. Polyakov introduced the concept of multi-normed space in their article [1]. Multi normed space has a relation with ordered vector spaces and operator spaces. Furthermore, this concept is somewhat similar to that of the operator sequence space. We have collected some properties of multi-normed spaces which will be used in this article. We refer readers to [1-4] for more details.

Functional equations and their stability are some of the classical and practical issues in the area of mathematical analysis. About half a century ago, the stability of functional equations was raised with the important question of Ulam [5]. It is said that a functional equation $G$ is stable if each function $g$ satisfying the equation $G$-approximately is near to the true solution of G. D. H. Hyers developed Ulam's question and theorem [6]. He posed the following theorem:

Suppose that $U$ and $V$ be Banach spaces and let $\rho$ be a function from $U$ to $V$ such that the following inequality satisfies for some $\delta>0$ and for every $u, v \in U$,

$$
\|\rho(u+v)-\rho(u)-\rho(v)\| \leq \delta .
$$

Then there is only one additive function $T: U \rightarrow V$ so that

$$
\|T(u)-\rho(u)\| \leq \delta
$$

for any $u \in U$.

Mathematicians developed the results of the Hyers theorem. By changing the space, the norm, the control function, and functional equation, they could prove more interesting theorems [7-14]. For example, the Jenson functional equation or the integral and differential equations were used instead of the functional equation (in the theorem) and the validity of the theorem was proved. Now, we change the functional equation to a different lattice functional equation and various control functions in the above theorem are replaced.

Definition 1. Let $X$ be a set. A function $d: X^{2} \rightarrow[0, \infty]$ is a called a generalized metric on $X$ if and only if $d$ satisfies 
$\left(M_{1}\right) d(x, y)=0$ if and only if $x=y$;

$\left(M_{2}\right) d(x, y)=d(y, x)$, for all $x, y \in X$;

$\left(M_{3}\right) d(x, z) \leq d(x, y)+d(y, z)$ for all $x, y, z \in X$.

We now introduce one of the fundamental results of the fixed point theory.

Theorem $1([15,16])$. Let $(X, d)$ be a generalized complete metric space. Assume that $G: X \rightarrow X$ is a strictly contractive operator with the Lipschitz constant $L<1$. If there exists a non-negative integer $n_{0}$ such that $d\left(G^{n_{0}+1} x, G^{n_{0}} x\right)<\infty$ for some $x \in X$, then the following statements are held:

(i) The sequence $\left\{G^{n} x\right\}$ converges to a fixed point $x_{0}$ of $G$;

(ii) $x_{0}$ is the unique fixed point of $G$ in $Y=\left\{y \in X \mid d\left(G^{n_{0}} x, y\right)<\infty\right\}$;

(iii) If $y \in Y$, then

$$
d\left(y, x_{0}\right) \leq \frac{1}{1-L} d(G y, y)
$$

Now, recall the notion of a multi-normed space from $[1,4]$. Let $(E,\|\|$.$) be a complex$ normed space and let $k \in \mathbb{N}$. We denote by $E^{k}$, the linear space $E \oplus \ldots \oplus E$ consisting of k-tuples $\left(x_{1}, \ldots, x_{k}\right)$, where $x_{1}, \ldots, x_{k} \in E$. The linear operations on $E^{k}$ are defined coordinatwise. The zero element of either $E$ or $E^{k}$ is denoted by 0 . We denote by $\mathbb{N}_{k}$ the set $\{1,2, \ldots, k\}$ and by $\mathcal{G}_{k}$ the group of permutations on $k$ symbols.

Definition 2. Let $(E,\|\|$.$) be a complex (real) normed space. A multi-normed on \left\{E^{k}, K \in \mathbb{N}\right\}$ is a sequence $\left\{\|.\|_{k}\right\}_{k \in \mathbb{N}}$ of norms on $E^{k}(k \in N)$ such that $\|x\|_{1}=\|x\|$, for each $x \in E$ and the following axioms are satisfied for each $k \in \mathbb{N}$ with $k \geq 2$ :

$\left(N_{1}\right)\left\|\left(x_{\sigma(1)}, \ldots, x_{\sigma(k)}\right)\right\|_{k}=\left\|\left(x_{1}, \ldots, x_{k}\right)\right\|_{k} \quad\left(\sigma \in \mathcal{G}_{k} ; x_{1}, \ldots, x_{k} \in E\right) ;$

$\left(N_{2}\right)\left\|\left(\alpha_{1} x_{1}, \ldots, \alpha_{k} x_{k}\right)\right\|_{k} \leq\left(\max _{i \in \mathbb{N}_{k}}\left|\alpha_{i}\right|\right)\left\|\left(x_{1}, \ldots, x_{k}\right)\right\|_{k}$

$\left(\alpha_{1}, \ldots, \alpha_{k} \in \mathbb{C} ; x_{1}, \ldots, x_{k} \in E\right)$;

$\left(N_{3}\right)\left\|\left(x_{1}, \ldots, x_{k-1}, 0\right)\right\|_{k}=\left\|\left(x_{1}, \ldots, x_{k-1}\right)\right\|_{k-1} \quad\left(x_{1}, \ldots, x_{k-1} \in E\right)$;

(N4) $\left\|\left(x_{1}, \ldots, x_{k-1}, x_{k-1}\right)\right\|_{k}=\left\|\left(x_{1}, \ldots, x_{k-1}\right)\right\|_{k-1} \quad\left(x_{1}, \ldots, x_{k-1} \in E\right)$.

In this case, we say that $\left\{\left(E^{k},\|.\|_{k}\right), k \in \mathbb{N}\right\}$ is a multi-normed space.

Suppose that $\left\{\left(E^{k},\|.\|_{k}\right), k \in \mathbb{N}\right\}$ is a multi-normed space. The following properties are almost immediate consequences of the axioms:

(i) $\|(x, \ldots, x)\|_{k}=\|x\| \quad(x \in E)$;

(ii) $\max _{i \in \mathbb{N}_{k}}|| x_{i}\|\leq\|\left(x_{1}, \ldots, x_{k}\right)\left\|_{k} \leq \sum_{i=1}^{k}\right\| x_{i}\left\|\leq k \max _{i \in \mathbb{N}_{k}}\right\| x_{i} \|$

$\left(x_{1}, \ldots, x_{k} \in E\right)$.

Applying (ii) one concludes that if $(E,\|\|$.$) is a Banach space, then \left(E^{K},\|\cdot\|_{k}\right)$ is a Banach space for each $k \in \mathbb{N}$; in this case, $\left\{\left(E^{k},\|.\|_{k}\right), k \in \mathbb{N}\right\}$ is called a multi-Banach space.

Definition 3. Let $(E,\|\|$.$) be a normed algebra such that \left\{\left(E^{K},\|\|.\right): k \in \mathbb{N}\right\}$ is a multi-normed space. Then $\left\{\left(E^{K},\|\|.\right): k \in \mathbb{N}\right\}$ is a multi-normed algebra if

$$
\left\|\left(x_{1} y_{1}, \ldots, x_{k} y_{k}\right)\right\| \leq\left\|\left(x_{1}, \ldots, x_{k}\right)\right\|_{k}\left\|\left(y_{1}, \ldots, y_{k}\right)\right\|_{k} \quad\left(\forall k \in \mathbb{N} \quad x_{1}, \ldots, x_{k}, y_{1}, \ldots, y_{k} \in E\right) .
$$

Furthermore, the multi-normed algebra $\left\{\left(E^{K},\|\|.\right): k \in \mathbb{N}\right\}$ is a multi-Banach algebra if $\left\{\left(E^{K},\|\|.\right): k \in \mathbb{N}\right\}$ is a multi-Banach space.

Definition 4 ([17]). Let $X$ be a real vector space. A function $N: X \times \mathbb{R} \rightarrow[0,1]$ is called a fuzzy norm on $X$ if for all $x, y \in X$ and all $s, t \in \mathbb{R}$

$\left(N_{1}\right) N(x, t)=0$, for all $t \leq 0$;

$\left(N_{2}\right) x=0$ if and only if $N(x, t)=1$ for all $t>0$;

$\left(N_{3}\right) N(c x, t)=N\left(x, \frac{t}{|c|}\right)$ if $c \neq 0$;

$\left(N_{4}\right) N(x+y, s+t) \geq \min \{N(x, s), N(y, t)\}$; 
$\left(N_{5}\right) N(x,$.$) is a non-decreasing function of \mathbb{R}$ and $\lim _{t \rightarrow \infty} N(x, t)=1$;

$\left(N_{6}\right)$ For $x \neq 0, N(x,$.$) is continuous on \mathbb{R}$.

The pair $(X, N)$ is called a fuzzy normed vector space.

Definition 5. Let $(X, N)$ be a fuzzy normed vector space.

(1) A sequence $\left\{x_{n}\right\}$ in $X$ is said to be convergent if there exists an $x \in X$ such that $\lim _{n \rightarrow \infty} N\left(x_{n}-\right.$ $x, t)=1, \quad \forall t>0$. In this case, $x$ is called the limit of the sequence $\left\{x_{n}\right\}$ and we denote it by $N-\lim _{n \rightarrow \infty} x_{n}=x$.

(2) A sequence $\left\{x_{n}\right\}$ in $X$ is called Cauchy if for each $\epsilon>0$ and each $t>0$ there exists an $n_{0} \in \mathbb{N}$ such that for all $n \geq n_{0}$ and all $p>0$, we have $N\left(x_{n+p}-x_{n}, t\right)>1-\epsilon$.

It is known that every convergent sequence in fuzzy normed space is Cauchy. If each Cauchy sequence is convergent, then the fuzzy norm is said to be complete and the fuzzy normed space is called a fuzzy Banach space. We say that a mapping $f: X \rightarrow Y$ between fuzzy normed vector spaces $X$ and $Y$ is continuous at a point $x_{0} \in X$ if for each sequence $\left\{x_{n}\right\}$ converging to $x_{0}$ in $X$, then the sequence $\left\{f\left(x_{n}\right)\right\}$ converges to $f\left(x_{0}\right)$. If $f: X \rightarrow Y$ is continuous at each $x_{0} \in X$, then $f: X \rightarrow Y$ is said to be continuous on $X$.

Definition 6 ([18]). Let $X$ be an algebra and $(X, N)$ a fuzzy normed space. The fuzzy normed space $(X, N)$ is called a fuzzy normed algebra if

$$
N\left(x x^{\prime}, s t\right) \geq N(x, s) N\left(x^{\prime}, t\right) \quad \forall x, x^{\prime} \in X, \quad s, t \in \mathbb{R}^{+} .
$$

Complete fuzzy normed algebra is called a fuzzy Banach algebra.

Example 1. Every normed algebra $(X,\|\|$.$) defines a fuzzy normed algebra (X, N)$, where $N$ is defined by

$$
N(x, t)=\frac{t}{t+\|x\|} \quad \forall x \in X, \quad \forall t>0 .
$$

This space is called the induced fuzzy normed algebra.

Now, we recall the notion of a multi-fuzzy normed space. The readers can consider [19] for more details about the features of this space.

Definition 7. Let $(E, N)$ be a fuzzy normed space. A multi-fuzzy norm on $\left\{E^{k}, k \in \mathbb{N}\right\}$ is a sequence $\left\{N_{k}\right\}$ such that $N_{k}$ is a fuzzy norm on $E^{k} \quad k \in \mathbb{N}, N_{1}(x, t)=N(x, t)$ for each $x \in E$ and $t \in \mathbb{R}$ and the following axioms are satisfied for each $k \in \mathbb{N}$ with $k \geq 2$ :

$\left(F_{1}\right) N_{k}\left(\left(x_{\sigma(1)}, \ldots, x_{\sigma(k)}\right), t\right)=N_{k}\left(\left(x_{1}, \ldots, x_{k}\right), t\right)$

$\left(\forall \sigma \in \mathcal{G}_{k}, \quad \forall x_{1}, \ldots, x_{k} \in E, \quad \forall t \in \mathbb{R}\right) ;$

$\left(F_{2}\right) N_{k}\left(\left(\alpha_{1} x_{1}, \ldots, \alpha_{k} x_{k}\right), t\right) \geq N_{k}\left(\max _{i \in \mathbb{N}_{k}}\left|\alpha_{i}\right|\left(x_{1}, \ldots, x_{k}\right), t\right)$

$\left(\forall \alpha_{1}, \ldots, \alpha_{k} \in \mathbb{C}, \quad \forall x_{1}, \ldots, x_{k} \in E, \forall t \in \mathbb{R}\right) ;$

$\left(F_{3}\right) N_{k}\left(\left(x_{1}, \ldots, x_{k-1}, 0\right), t\right)=N_{k-1}\left(\left(x_{1}, \ldots, x_{k-1}\right), t\right)$

$\left(\forall x_{1}, \ldots, x_{k-1} \in E, \quad \forall t \in \mathbb{R}\right)$;

(F4) $N_{k}\left(\left(x_{1}, \ldots, x_{k-1}, x_{k-1}\right), t\right)=N_{k-1}\left(\left(x_{1}, \ldots, x_{k-1}\right), t\right)$

$\left(\forall x_{1}, \ldots, x_{k-1} \in E, \quad \forall t \in \mathbb{R}\right)$.

In this case, we say that $\left\{\left(E^{k}, N_{k}\right), k \in N\right\}$ is a multi-fuzzy normed space.

If $\left(E, N_{1}\right)$ is a fuzzy Banach space, then $\left\{\left(E^{k}, N_{k}\right), k \in N\right\}$ is a multi-fuzzy Banach space (see [19]).

\section{Main Result}

We begin this section by introducing the notion of multi-fuzzy normed algebra. Then we develop the subject of the article [20] in multi-fuzzy Banach algebras. 
Definition 8. Let (E,N) be a fuzzy normed algebra, and let $\left\{\left(E^{k}, N_{k}\right), k \in N\right\}$ be a multi-fuzzy normed space. Then $\left\{\left(E^{k}, N_{k}\right), k \in \mathbb{N}\right\}$ is a multi-fuzzy normed algebra if

$$
N_{k}\left(\left(x_{1} y_{1}, \ldots, x_{k} y_{k}\right), s t\right) \geq N_{k}\left(\left(x_{1}, \ldots, x_{k}\right), s\right) N_{k}\left(\left(y_{1}, \ldots, y_{k}\right), t\right),
$$

for all $k \in \mathbb{N}, x_{1}, \ldots, x_{k}, y_{1}, \ldots, y_{k} \in E$ and $s, t \in \mathbb{R}^{+}$. Furthermore, the multi-fuzzy normed algebra $\left\{\left(E^{k}, N_{k}\right), k \in N\right\}$ is a multi-fuzzy Banach algebra if $\left\{\left(E^{k}, N_{k}\right), k \in N\right\}$ is a multi-fuzzy Banach space.

Example 2. Every multi-Banach algebra $\left\{\left(E^{k},\|.\|_{k}\right), k \in N\right\}$ defines a multi-fuzzy Banach algebra $\left\{\left(E^{k}, N_{k}\right), k \in N\right\}$, where

$$
N_{k}\left(\left(x_{1}, \ldots, x_{k}\right), t\right)=\frac{t}{t+\left\|\left(x_{1}, \ldots, x_{k}\right)\right\|_{k}} \quad t \in \mathbb{R}^{+}, \quad x_{1}, \ldots, x_{k} \in E .
$$

In this article, we assume that $m_{0}$ is a natural number. We also assume that $\mathbb{T}^{1}=\{z \in$ $\mathbb{C}:|z|=1\}$ and $\mathbb{T}_{\frac{1}{m_{0}}}^{1}:=\left\{e^{i \theta} ; 0 \leq \theta \leq \frac{2 \pi}{m_{0}}\right\}$. Moreover, we suppose that $(E, N)$ is fuzzy Banach algebra. For a given mapping $f: E \rightarrow E$, we define

$$
D_{\lambda \gamma} f(x, y)=\bar{\lambda} f\left(\frac{x+\gamma y}{2}\right)+\bar{\lambda} f\left(\frac{x-\gamma y}{2}\right)-f(\lambda x) \quad \forall x, y \in E \quad \text { and } \quad \forall \lambda, \gamma \in \mathbb{C} .
$$

Let us recall some of the necessary definitions.

Let $A$ be an algebra over $\mathbb{C}$. An involution on $A$ is a mapping

$$
\begin{aligned}
\star: A & \rightarrow A \\
a & \longmapsto a^{\star}
\end{aligned}
$$

such that

(i) $(\alpha a+\beta b)^{\star}=\bar{\alpha} a^{\star}+\bar{\beta} b^{\star} \quad \forall a, b \in A, \quad \forall \alpha, \beta \in \mathbb{C}$;

(ii) $(a b)^{\star}=b^{\star} a^{\star} \quad \forall a, b \in A$;

(iii) $a^{\star \star}=a \quad \forall a \in A$.

1. A complex algebra with an involution is a $\star$-algebra.

2. A $C^{\star}$-algebras is a (non-zero) Banach algebra with an involution, such that:

$$
\left\|a^{\star} a\right\|=\|a\|^{2} .
$$

Definition 9. Let $A$ be an $\star$-algebra and $(A, N)$ a fuzzy normed algebra. The fuzzy normed algebra $(A, N)$ is called a fuzzy normed $\star$-algebra if

$$
N\left(a^{\star}, t\right)=N(a, t) \quad \forall a \in A, \quad \forall t \in \mathbb{R}^{+} .
$$

A complete fuzzy normed $*$-algebra is called a fuzzy Banach $*$-algebra.

Definition 10. Let $(A, N)$ be a fuzzy Banach $*$-algebra. Then $(A, N)$ is called a fuzzy $C^{*}$-algebra if

$$
N\left(a^{\star} a, s t\right)=N\left(a^{\star}, s\right) N(a, t) \quad \forall a \in A, \quad \forall s, t \in \mathbb{R}^{+} .
$$

Theorem 2. Let $(E, N)$ be a fuzzy Banach algebra and $\left\{\left(E^{K}, N_{k}\right), k \in \mathbb{N}\right\}$ be a multi-fuzzy Banach algebra. In addition, suppose that $\psi: E^{2 k} \rightarrow[0, \infty)$ is a given function and there exists a constant $L, 0<L<1$, such that:

$$
\begin{gathered}
\psi\left(x_{1}, y_{1}, \ldots, x_{k}, y_{k}\right) \leq 2 L \psi\left(\frac{x_{1}}{2}, \frac{y_{1}}{2}, \ldots, \frac{x_{k}}{2}, \frac{y_{k}}{2}\right), \\
N_{k}\left(\left(D_{\lambda \gamma} f\left(x_{1}, y_{1}\right), D_{\lambda \gamma} f\left(x_{2}, y_{2}\right), \ldots, D_{\lambda \gamma} f\left(x_{k}, y_{k}\right)\right), t\right) \geq \frac{t}{t+\psi\left(x_{1}, y_{1}, \ldots, x_{k}, y_{k}\right)},
\end{gathered}
$$




$$
\begin{gathered}
N_{k}\left(\left(f\left(x_{1} y_{1}\right)-f\left(y_{1}\right) f\left(x_{1}\right), \ldots, f\left(x_{k} y_{k}\right)-f\left(y_{k}\right) f\left(x_{k}\right)\right), t\right) \geq \frac{t}{t+\psi\left(x_{1}, y_{1}, \ldots, x_{k}, y_{k}\right)}, \\
N-\lim _{n \rightarrow \infty} 2^{-n} f\left(2^{n}\left(N-\lim _{n \rightarrow \infty} 2^{-n} f\left(2^{n} x\right)\right)\right)=x,
\end{gathered}
$$

for all $x_{1}, \ldots, x_{k}, y_{1}, \ldots, y_{k} \in E$, all $t>0$ and all $\lambda, \gamma \in \mathbb{T}_{\frac{1}{m_{0}}}^{1}$, then there exists a unique involution $H: E \rightarrow$ E such that

$$
H(x):=N-\lim _{n \rightarrow \infty} 2^{-n} f\left(2^{n} x\right)
$$

and

$$
N_{k}\left(\left(f\left(x_{1}\right)-H\left(x_{1}\right), \ldots, f\left(x_{k}\right)-H\left(x_{k}\right)\right), t\right) \geq \frac{(1-L) t}{(1-L) t+L \psi\left(x_{1}, 0, \ldots, x_{k}, 0\right)} .
$$

Also, if for all $x_{1}, \ldots, x_{k} \in E$ and for all $t>0$

$$
N_{k}\left(\left(\left[N\left(f\left(x_{1}\right), t\right)-N\left(x_{1}, t\right)\right] x_{1}, \ldots,\left[N\left(f\left(x_{k}\right), t\right)-N\left(x_{k}, t\right)\right] x_{k}\right), t\right) \geq \frac{t}{t+\psi\left(x_{1}, x_{1}, \ldots, x_{k}, x_{k}\right)},
$$

then $(E, N)$ is a fuzzy Banach $*$-algebra.

Moreover, if for all $x_{1}, \ldots, x_{k} \in E$ and for all $s, t>0$

$$
\begin{aligned}
& N_{k}\left(\left(\left[N\left(f\left(x_{1}\right) x_{1}, s t\right)-N\left(f\left(x_{1}\right), s\right) N\left(x_{1}, t\right)\right] x_{1}, \ldots,\right.\right. \\
& \left.\left.\left[N\left(f\left(x_{k}\right) x_{k}, s t\right)-N\left(f\left(x_{k}\right), s\right) N\left(x_{k}, t\right)\right] x_{k}\right), t\right) \geq \frac{t}{t+\psi\left(x_{1}, x_{1}, \ldots, x_{k}, x_{k}\right)},
\end{aligned}
$$

then $(E, N)$ is a fuzzy $C^{*}$-algebra with involution $x^{*}=H(x)$ for all $x \in E$.

Proof. Consider the set $S:=\{g: E \rightarrow E\}$ and introduce the generalized metric on $S$.

$d(g, h)=\inf \left\{\delta \in[0, \infty]: N_{k}\left(\left(g\left(x_{1}\right)-h\left(x_{1}\right), \ldots, g\left(x_{k}\right)-h\left(x_{k}\right)\right), \delta t\right) \geq \frac{t}{t+\psi\left(x_{1}, 0, \ldots, x_{k}, 0\right)}\right\}$, for all $x_{1}, \ldots, x_{k} \in E$ and $t>0$. Where, as usual, $\inf \varnothing=+\infty$. It is easy to show that $(S, d)$ is complete (see [21]). Now, we define mappings $J: S \rightarrow S$ by

$$
J g(x):=\frac{1}{2} g(2 x) \quad \forall x \in E .
$$

First, we prove that $J$ is strictly contractive on $S$. Let $g, h \in S$ be given such that $d(g, h) \neq+\infty$. Then for some $\epsilon>0$

$$
N_{k}\left(\left(g\left(x_{1}\right)-h\left(x_{k}\right), \ldots, g\left(x_{k}\right)-h\left(x_{k}\right)\right), \epsilon t\right) \geq \frac{t}{t+\psi\left(x_{1}, 0, \ldots, x_{k}, 0\right)} \quad \forall x_{1}, \ldots, x_{k} \in E, \forall t>0 .
$$

If we replace $x_{k}$ in the above inequality with $2 x_{k}$, for $k=1, \ldots, n$, and make use of (2), then we have

$$
\begin{aligned}
N & \left(\left(J g\left(x_{1}\right)-J h\left(x_{1}\right), \ldots, J g\left(x_{k}\right)-J h\left(x_{k}\right)\right), L \epsilon t\right) \\
& =N\left(\left(\frac{1}{2} g\left(2 x_{1}\right)-\frac{1}{2} h\left(2 x_{1}\right), \ldots, \frac{1}{2} g\left(2 x_{k}\right)-\frac{1}{2} h\left(2 x_{k}\right)\right), L \epsilon t\right) \\
& =N\left(\left(g\left(2 x_{1}\right)-h\left(2 x_{1}\right), \ldots, g\left(2 x_{k}\right)-h\left(2 x_{k}\right)\right), 2 L \epsilon t\right) \\
& \geq \frac{2 L t}{2 L t+\psi\left(2 x_{1}, 0, \ldots, 2 x_{k}, 0\right)} \\
& \geq \frac{2 L t}{2 L t+2 L \psi\left(x_{1}, 0, \ldots, x_{k}, 0\right)}=\frac{t}{t+\psi\left(x_{1}, 0, \ldots, x_{k}, 0\right)},
\end{aligned}
$$


for all $x_{1}, \ldots, x_{k} \in E$ and all $t>0$. Therefore, using the definition of $\mathrm{d}$ metric, we can conclude that $d(J g, J h) \leq L \epsilon$. This means that

$$
d(J g, J h) \leq L d(g, h) \quad \forall g, h \in S .
$$

Next, we assert that $d(J f, f)<\infty$. Putting $\lambda=1$ and $y_{1}=\ldots=y_{k}=0$ in (3), we get

$$
\begin{aligned}
& N_{k}\left(\left(\frac{1}{2} f\left(2 x_{1}\right)-f\left(x_{1}\right), \ldots, \frac{1}{2} f\left(2 x_{k}\right)-f\left(x_{k}\right)\right), L t\right) \\
& \quad=N_{k}\left(\left(f\left(2 x_{1}\right)-2 f\left(x_{1}\right), \ldots, f\left(2 x_{k}\right)-2 f\left(x_{k}\right)\right), 2 L t\right) \\
& \quad \geq \frac{2 L t}{2 L t+\psi\left(2 x_{1}, 0, \ldots, 2 x_{k}, 0\right)} \\
& \quad \geq \frac{2 L t}{2 L t+2 L \psi\left(x_{1}, 0, \ldots, x_{k}, 0\right)}=\frac{t}{t+\psi\left(x_{1}, 0, \ldots, x_{k}, 0\right)},
\end{aligned}
$$

for any $x_{1}, \ldots, x_{k} \in E$, that is

$$
d(J f, f) \leq L<\infty .
$$

Now, it follows Theorem 1 that there exists a function $H: E \rightarrow E$ which is a fixed point of $J$, i.e,

$$
H(x)=\frac{1}{2} H(2 x),
$$

such that $\lim _{n \rightarrow \infty} d\left(J^{n} f, H\right)=0$. Therefore, it can be concluded that

$$
N-\lim _{n \rightarrow \infty} \frac{1}{2^{n}} f\left(2^{n} x\right)=H(x) \quad \forall x \in E
$$

Then $H \in X^{*}$, which:

$$
X^{*}=\{g \in S: d(f, g)<\infty\} .
$$

Again, by Theorem 1 and (10), we obtain

$$
d(f, H) \leq \frac{1}{1-L} d(J f, f) \leq \frac{L}{1-L},
$$

i.e, the inequality (6) is true for all $x \in E$. Suppose $\lambda=\gamma=1$ in (2), we have

$$
\begin{aligned}
& N_{k}\left(\left(2^{-n} f\left(2^{n}\left(\frac{x_{1}+y_{1}}{2}\right)\right)+2^{-n} f\left(2^{n}\left(\frac{x_{1}-y_{1}}{2}\right)\right)-2^{-n} f\left(2^{n} x_{1}\right), \ldots,\right.\right. \\
& \left.\left.2^{-n} f\left(2^{n}\left(\frac{x_{k}+y_{k}}{2}\right)\right)+2^{-n} f\left(2^{n}\left(\frac{x_{k}-y_{k}}{2}\right)\right)-2^{-n} f\left(2^{n} x_{k}\right)\right), 2^{-n} t\right) \\
& \geq \frac{t}{t+\psi\left(2^{n} x_{1}, 2^{n} y_{1}, \ldots, 2^{n} x_{k}, \ldots, 2^{n} y_{k}\right)} .
\end{aligned}
$$

Thus,

$$
\begin{aligned}
& N_{k}\left(\left(2^{-n} f\left(2^{n}\left(\frac{x_{1}+y_{1}}{2}\right)\right)+2^{-n} f\left(2^{n}\left(\frac{x_{1}-y_{1}}{2}\right)\right)-2^{-n} f\left(2^{n} x_{1}\right), \ldots,\right.\right. \\
& \left.2^{-n} f\left(2^{n}\left(\frac{x_{k}+y_{k}}{2}\right)\right)+2^{-n} f\left(2^{n}\left(\frac{x_{k}-y_{k}}{2}\right)\right)-2^{-n} f\left(2^{n} x_{k}\right), t\right) \\
& \geq \frac{2^{n} t}{2^{n} t+2^{n} L^{n} \psi\left(x_{1}, y_{1}, \ldots, x_{k}, \ldots, y_{k}\right)} \rightarrow 1 \text { as } n \rightarrow \infty,
\end{aligned}
$$

for all $x_{1}, . . x_{k}, y_{1}, \ldots, y_{k}$ in $E$ and for all $t>0$. Therefore

$N_{k}\left(\left(H\left(\frac{x_{1}+y_{1}}{2}\right)+H\left(\frac{x_{1}-y_{1}}{2}\right)-H\left(x_{1}\right), \ldots, H\left(\frac{x_{k}+y_{k}}{2}\right)+H\left(\frac{x_{k}-y_{k}}{2}\right)-H\left(x_{k}\right)\right), t\right)=1$. 
By replacing $x_{1}, \ldots, x_{k}$ with $x$ and $y_{1}, \ldots, y_{k}$ with $y$ in the last inequality, we conclude

$$
N\left(H\left(\frac{x+y}{2}\right)+H\left(\frac{x-y}{2}\right)-H(x), t\right)=1 .
$$

We get

$$
H(x)=H\left(\frac{x+y}{2}\right)+H\left(\frac{x-y}{2}\right)
$$

for all $x, y \in E$. If $y_{1}=\ldots=y_{k}=0$ in (3), then we have

$$
\begin{aligned}
& N_{k}\left(\left(2^{-n} \bar{\lambda} f\left(2^{n-1} x_{1}\right)+2^{-n} \bar{\lambda} f\left(2^{n-1} x_{1}\right)-2^{-n} f\left(\lambda 2^{n} x_{1}\right), \ldots,\right.\right. \\
& \left.\left.\quad 2^{-n} \bar{\lambda} f\left(2^{n-1} x_{k}\right)+2^{-n} \bar{\lambda} f\left(2^{n-1} x_{k}\right)-2^{-n} f\left(\lambda 2^{n} x_{k}\right)\right), 2^{-n} t\right) \\
& \quad \geq \frac{t}{t+\psi\left(2^{n} x_{1}, 0, \ldots, 2^{n} x_{k}, \ldots, 0\right)} .
\end{aligned}
$$

Thus,

$$
\begin{aligned}
& N_{k}\left(\left(2^{-n} \bar{\lambda} f\left(2^{n-1} x_{1}\right)+2^{-n} \bar{\lambda} f\left(2^{n-1} x_{1}\right)-2^{-n} f\left(\lambda 2^{n} x_{1}\right), \ldots,\right.\right. \\
& \left.\left.2^{-n} \bar{\lambda} f\left(2^{n-1} x_{k}\right)+2^{-n} \bar{\lambda} f\left(2^{n-1} x_{k}\right)-2^{-n} f\left(\lambda 2^{n} x_{k}\right)\right), t\right) \\
& \geq \frac{2^{n} t}{2^{n} t+2^{n} L^{n} \psi\left(x_{1}, 0, \ldots, x_{k}, \ldots, 0\right)} \rightarrow 1 \text { as } n \rightarrow \infty,
\end{aligned}
$$

for all $x_{1}, \ldots, x_{k} \in E$ and $t>0$, then

$$
\begin{gathered}
N_{k}\left(\left(2 \bar{\lambda} H\left(\frac{x_{1}}{2}\right)-H\left(\lambda x_{1}\right), \ldots, 2 \bar{\lambda} H\left(\frac{x_{k}}{2}\right)-H\left(\lambda x_{k}\right)\right), t\right)=1, \\
\forall x_{1}, . ., x_{k} \in E, \quad \forall \lambda \in \mathbb{T}_{\frac{1}{m_{0}}}^{1}, \quad \forall t>0 .
\end{gathered}
$$

By replacing $x_{1}, \ldots, x_{k}$ with $x$ in the last inequality, we conclude

$$
N\left(\left(2 \bar{\lambda} H\left(\frac{x}{2}\right)-H(\lambda x), t\right)=1 \quad \forall x_{1}, . ., x_{k} \in E, \quad \forall \lambda \in \mathbb{T}_{\frac{1}{m_{0}}}^{1}, \quad \forall t>0 .\right.
$$

It follows by the last equation and additivity of $H$ that $H(\lambda x)=\bar{\lambda} H(x)$, for all $x \in E$ and all $\lambda \in \mathbb{T}_{\frac{1}{m_{0}}}^{1}$.

We will use techniques [22] to continue proving. Now, we show that $H$ is conjugate linear. We have to show that $H(\alpha x)=\bar{\alpha} H(x)$ for all $\alpha \in \mathbb{C}, x \in E$. To this end, let $\alpha \in \mathbb{C}$. If $\alpha$ belongs to $\mathbb{T}^{1}$, then there exists $\theta \in[0,2 \pi]$ such that $\alpha=e^{i \theta}$. We set $\alpha_{1}=e^{\frac{i \theta}{m_{0}}}$, thus $\alpha_{1}$ belongs to $\mathbb{T}_{\frac{1}{m_{0}}}^{1}$ and $H(\alpha x)=H\left(\alpha_{1}^{m_{0}} x\right)=\bar{\alpha}_{1}^{m_{0}} H(x)=\bar{\alpha} H(x)$.

If $\alpha$ belong to $n \mathbb{T}^{1}=\left\{n z ; \quad z \in \mathbb{T}^{1}\right\}$ for some $n \in \mathbb{N}$, then by additivity of $H$, $H(\alpha x)=\bar{\alpha} H(x)$ for all $x \in E$.

Let $t \in(0, \infty)$ then by Archimedes property of $\mathbb{C}$, there exists a positive real number $n$ such that the point $(t, 0)$ lies in the interior of a circle with centre at origin and radius $n$. Putting $t_{1}:=t+\sqrt{n^{2}-t^{2}} i, t_{2}:=t-\sqrt{n^{2}-t^{2}} i$. Then we have $t=\frac{t_{1}+t_{2}}{2}$ and $t_{1}, t_{2} \in n \mathbb{T}^{1}$. It follows that

$$
H(t x)=H\left(\frac{t_{1}+t_{2}}{2} x\right)=\frac{\bar{t}_{1}}{2} H(x)+\frac{\bar{t}_{2}}{2} H(x)=\bar{t} H(x)=t H(x) \quad \forall x \in E .
$$


On the other hand, there exists $\theta \in[0,2 \pi]$ such that $\alpha=|\alpha| e^{i \theta}$. It follows that

$$
H(\alpha x)=H\left(|\alpha| e^{i \theta} x\right)=|\alpha| e^{-i \theta} H(x)=\bar{\alpha} H(x) \quad \forall x \in E .
$$

Hence, $H: E \rightarrow E$ is conjugate $\mathbb{C}$-linear mapping. By (4)

$$
\begin{aligned}
& N_{k}\left(\left(4^{-n} f\left(4^{n} x_{1} y_{1}\right)-2^{-n} f\left(2^{n} y_{1}\right) \cdot 2^{-n} f\left(2^{n} x_{1}\right), \ldots,\right.\right. \\
& \left.\left.4^{-n} f\left(4^{n} x_{k} y_{k}\right)-2^{-n} f\left(2^{n} y_{k}\right) \cdot 2^{-n} f\left(2^{n} x_{k}\right)\right), 4^{-n} t\right) \\
& \geq \frac{t}{t+\psi\left(2^{n} x_{1}, 2^{n} y_{1}, \ldots, 2^{n} x_{k}, 2^{n} y_{k}\right)} x \\
& \geq \frac{t}{t+2^{n} L^{n} \psi\left(x_{1}, y_{1}, \ldots, x_{k}, y_{k}\right)}
\end{aligned}
$$

thus

$$
\begin{gathered}
N_{k}\left(\left(4^{-n} f\left(4^{n} x_{1} y_{1}\right)-2^{-n} f\left(2^{n} y_{1}\right) \cdot 2^{-n} f\left(2^{n} x_{1}\right), \ldots,\right.\right. \\
\left.\left.4^{-n} f\left(4^{n} x_{k} y_{k}\right)-2^{-n} f\left(2^{n} y_{k}\right) \cdot 2^{-n} f\left(2^{n} x_{k}\right)\right), t\right) \\
\geq \frac{4^{n} t}{4^{n} t+2^{n} L^{n} \psi\left(x_{1}, y_{1}, \ldots, x_{k}, y_{k}\right)} \rightarrow 1 \text { as } n \rightarrow \infty,
\end{gathered}
$$

for all $x_{1}, \ldots, x_{k}, y_{1}, \ldots, y_{k} \in E$ and all $t>0$, so we have

$$
\begin{aligned}
N_{k}\left(\left(H\left(x_{1} y_{1}\right)-H\left(y_{1}\right) H\left(x_{1}\right), \ldots, H\left(x_{k} y_{k}\right)-H\left(y_{k}\right) H\left(x_{k}\right)\right), t\right)=1 \\
\forall x_{1}, \ldots, x_{k}, y_{1}, \ldots, y_{k} \in E, \forall t>0
\end{aligned}
$$

By replacing $x_{1}, \ldots, x_{k}$ with $x$ and $y_{1}, \ldots, y_{k}$ with $y$ in the last inequality, we conclude

$$
N(H(x y)-H(y) H(x), t)=1 \quad \forall x, y \in E, \quad \forall t>0
$$

and therefore,

$$
H(x y)=H(y) H(x) \quad \forall x, y \in E .
$$

On the other hand, by (5)

$$
H(H(x))=N-\lim _{n \rightarrow \infty} 2^{-n} f\left(2^{n}\left(N-\lim _{k \rightarrow \infty} 2^{-n} f\left(2^{n} x\right)\right)\right)=x,
$$

for all $x$ in $E$. Hence $H: E \rightarrow E$ is an involution satisfying (6).

In addition, we must prove the uniqueness of $H$. In fact, assume the existence of another involution $H^{\prime}$ satisfies (6), hence $H^{\prime}\left(\frac{x}{2^{k}}\right)=\frac{1}{2^{k}} H^{\prime}(x), \forall x \in E$, so we have

$$
\begin{aligned}
& N_{k}\left(\left(2^{-n} f\left(2^{n} x_{1}\right)-2^{-n} H^{\prime}\left(2^{n} x_{1}\right), \ldots, 2^{-n} f\left(2^{n} x_{k}\right)-2^{-n} H^{\prime}\left(2^{n} x_{k}\right)\right), t\right) \\
& =N\left(\left(f\left(2^{n} x_{1}\right)-H^{\prime}\left(2^{n} x_{1}\right), \ldots, f\left(2^{n} x_{k}\right)-H^{\prime}\left(2^{n} x_{k}\right)\right), 2^{n} t\right) \\
& \geq \frac{(1-L) 2^{n} t}{(1-L) 2^{n} t+L \psi\left(2^{n} x_{1}, 0, \ldots, 2^{n} x_{k}, 0\right)} \\
& =\frac{(1-L) 2^{n} t}{(1-L) 2^{n} t+2^{n} L^{n+1} \psi\left(x_{1}, 0, \ldots, x_{k}, 0\right)} \rightarrow 1 \text { as } n \rightarrow \infty
\end{aligned}
$$

for all $x_{1}, \ldots, x_{k} \in E, t>0$, then

$$
N_{k}\left(\left(H\left(x_{1}\right)-H^{\prime}\left(x_{1}\right), \ldots, H\left(x_{k}\right)-H^{\prime}\left(x_{k}\right)\right), t\right)=1 \quad \forall x_{1}, \ldots, x_{k} \in E, \quad \forall t>0
$$


By replacing $x_{1}, \ldots, x_{k}$ with $x$ in the last inequality, we conclude

$$
N\left(H(x)-H^{\prime}(x), t\right)=1 \quad \forall x \in E, \quad \forall t>0
$$

Therefore, $H(x)=H^{\prime}(x)$ for all $x \in E$. Now, suppose that $H$ satisfies (7), then we have

$$
\begin{aligned}
& N_{k}\left(\left(\left[N\left(2^{-n} f\left(2^{n} x_{1}\right), 2^{-n} t\right)-N\left(x_{1}, 2^{-n} t\right)\right] x_{1}, \ldots,\right.\right. \\
& \left.\left.\left[N\left(2^{-n} f\left(2^{n} x_{k}\right), 2^{-n} t\right)-N\left(x_{k}, 2^{-n} t\right)\right] x_{k}\right), 2^{-n} t\right) \\
& \geq \frac{t}{t+\psi\left(2^{n} x_{1}, 2^{n} x_{1}, \ldots, 2^{n} x_{k}, 2^{n} x_{k}\right)},
\end{aligned}
$$

thus,

$$
\begin{aligned}
& N_{k}\left(\left(\left[N\left(2^{-n} f\left(2^{n} x_{1}\right), t\right)-N\left(x_{1}, t\right)\right] x_{1}, \ldots,\right.\right. \\
& \left.\left.\quad\left[N\left(2^{-n} f\left(2^{n} x_{k}\right), t\right)-N\left(x_{k}, t\right)\right] x_{k}\right), t\right) \\
& \quad \geq \frac{2^{n} t}{2^{n} t+2^{n} L^{n} \psi\left(x_{1}, x_{1}, \ldots, x_{k}, x_{k}\right)} \rightarrow 1 \text { as } n \rightarrow \infty,
\end{aligned}
$$

for all $x_{1}, \ldots, x_{k} \in E$ and $t>0$, therefore

$$
N_{k}\left(\left(\left[N\left(H\left(x_{1}\right), t\right)-N\left(x_{1}, t\right)\right] x_{1}, \ldots,\left[N\left(H\left(x_{k}\right), t\right)-N\left(x_{k}, t\right)\right] x_{k}\right), t\right)=1
$$

for all $x_{1}, \ldots, x_{k} \in E$ and $t>0$. Putting $x_{1}=\ldots=x_{k}:=x$ in the above equality, we get

$$
\begin{aligned}
& N([N(H(x), t)-N(x, t)] x, t)=1 \quad \forall x \in E, \quad t>0 \\
& \Longrightarrow[N(H(x), t)-N(x, t)] x=0 \quad \forall x \in E, \quad t>0 \\
& \Longrightarrow N(H(x), t)-N(x, t)=0 \quad \forall x \in E, \quad t>0
\end{aligned}
$$

Therefore, $N(H(x), t)=N(x, t)$ and $(E, N)$ is a fuzzy Banach $*$-algebra. Finally, we assume that $H$ satisfies (8). Then we have

$$
\begin{aligned}
& N_{k}\left(\left(\left[N\left(2^{-n} f\left(2^{n} x_{1}\right) x_{1}, 2^{-2 n} s t\right)-N\left(2^{-n} f\left(2^{n} x_{1}\right), 2^{-n} s\right) N\left(x_{1}, 2^{-n} t\right)\right] x_{1}, \ldots\right.\right. \\
& \left.\left.\left[N\left(2^{-n} f\left(2^{n} x_{k}\right) x_{k}, 2^{-2 n} s t\right)-N\left(2^{-n} f\left(2^{n} x_{k}\right), 2^{-n} s\right) N\left(x_{k}, 2^{-n} t\right)\right] x_{k}\right), 2^{-n} t\right) \\
& \geq \frac{t}{t+\psi\left(2^{n} x_{1}, 2^{n} x_{1}, \ldots, 2^{n} x_{k}, \ldots, 2^{n} x_{k}\right)}
\end{aligned}
$$

Thus,

$$
\begin{aligned}
& N_{k}\left(\left(\left[N\left(2^{-n} f\left(2^{n} x_{1}\right) x_{1}, s t\right)-N\left(2^{-n} f\left(2^{n} x_{1}\right), s\right) N\left(x_{1}, t\right)\right] x_{1}, \ldots,\right.\right. \\
& \left.\left.\left[N\left(2^{-n} f\left(2^{n} x_{k}\right) x_{k}, s t\right)-N\left(2^{-n} f\left(2^{n} x_{k}\right), s\right) N\left(x_{k}, t\right)\right] x_{k}\right), t\right) \\
& \geq \frac{2^{n} t}{2^{n} t+2^{n} L^{n} \psi\left(x_{1}, x_{1}, \ldots, x_{k}, \ldots, x_{k}\right)} .
\end{aligned}
$$

Again, similarly to the above it can be concluded 


$$
\begin{aligned}
& N_{k}\left(\left(\left[N\left(H\left(x_{1}\right) x_{1}, s t\right)-N\left(H\left(x_{1}\right), s\right) N\left(x_{1}, t\right)\right] x_{1}, \ldots,\right.\right. \\
& {\left.\left.\left[N\left(H\left(x_{k}\right) x_{k}, s t\right)-N\left(H\left(x_{k}\right), s\right) N\left(x_{k}, t\right)\right] x_{k}\right), t\right)=1 \quad \forall x_{1}, \ldots, x_{k} \in E, \quad s, t>0 } \\
& \Longrightarrow \quad N([N(H(x) x, s t)-N(H(x), s) N(x, t)] x, t)=1 \quad \forall x \in E, \quad s, t>0 \\
& \Longrightarrow \quad[N(H(x) x, s t)-N(H(x), s) N(x, t)] x=0 \quad \forall x \in E, \quad s, t>0 \\
& \Longrightarrow \quad N(H(x) x, t)-N(H(x), s) N(x, t)=0 . \quad \forall x \in E, \quad s, t>0 .
\end{aligned}
$$

Therefore, $N(H(x) x, s t)=N(H(x), s) N(x, t)$, then $E$ is a $C^{*}$-algebra with involution $x^{*}=$ $H(x)$, for all $x \in E$.

Theorem 3. Let $(E, N)$ be a fuzzy Banach algebra and $\left\{\left(E^{K}, N_{k}\right), k \in \mathbb{N}\right\}$ be a multi-fuzzy Banach algebra. In addition, suppose that $\psi: E^{2 k} \rightarrow[0, \infty)$ is a given function and there exists a constant $L, 0<L<\frac{1}{2}$, such that

$$
\begin{gathered}
\psi\left(x_{1}, y_{1}, \ldots, x_{k}, y_{k}\right) \leq \frac{L}{2} \psi\left(2 x_{1}, 2 y_{1}, \ldots, 2 x_{k}, 2 y_{k}\right), \\
N_{k}\left(\left(D_{\lambda \gamma} f\left(x_{1}, y_{1}\right), D_{\lambda \gamma} f\left(x_{2}, y_{2}\right), \ldots, D_{\lambda \gamma} f\left(x_{k}, y_{k}\right)\right), t\right) \geq \frac{t}{t+\psi\left(x_{1}, y_{1}, \ldots, x_{k}, y_{k}\right)}, \\
N_{k}\left(\left(f\left(x_{1} y_{1}\right)-f\left(y_{1}\right) f\left(x_{1}\right), \ldots, f\left(x_{k} y_{k}\right)-f\left(y_{k}\right) f\left(x_{k}\right)\right), t\right) \geq \frac{t}{t+\psi\left(x_{1}, y_{1}, \ldots, x_{k}, y_{k}\right)}, \\
N-\lim _{n \rightarrow \infty} 2^{n} f\left(2^{-n}\left(N-\lim _{n \rightarrow \infty} 2^{n} f\left(2^{-n} x\right)\right)\right)=x,
\end{gathered}
$$

for all $x_{1}, \ldots, x_{k}, y_{1}, \ldots, y_{k} \in E$, all $t>0$ and all $\lambda, \gamma \in \mathbb{T}_{\frac{1}{m_{0}}}^{1}$, then there exists a unique involution $H: E \rightarrow$ Esuch that

$$
H(x):=N-\lim _{n \rightarrow \infty} 2^{n} f\left(2^{-n} x\right)
$$

and

$$
N_{k}\left(\left(f\left(x_{1}\right)-H\left(x_{1}\right), \ldots, f\left(x_{k}\right)-H\left(x_{k}\right)\right), t\right) \geq \frac{(1-L) t}{(1-L) t+\psi\left(x_{1}, 0, \ldots, x_{k}, 0\right)} .
$$

Further, if for all $x_{1}, \ldots, x_{k} \in E$ and for all $t>0$

$$
N_{k}\left(\left(\left[N\left(f\left(x_{1}\right), t\right)-N\left(x_{1}, t\right)\right] x_{1}, \ldots,\left[N\left(f\left(x_{k}\right), t\right)-N\left(x_{k}, t\right)\right] x_{k}\right), t\right) \geq \frac{t}{t+\psi\left(x_{1}, x_{1}, \ldots, x_{k}, x_{k}\right)},
$$

then $(E, N)$ is a fuzzy Banach *-algebra.

Moreover, if for all $x_{1}, \ldots, x_{k} \in E$ and for all $s, t>0$

$$
\begin{aligned}
& N_{k}\left(\left(\left[N\left(f\left(x_{1}\right) x_{1}, s t\right)-N\left(f\left(x_{1}\right), s\right) N\left(x_{1}, t\right)\right] x_{1}, \ldots,\right.\right. \\
& \left.\left.\left[N\left(f\left(x_{k}\right) x_{k}, s t\right)-N\left(f\left(x_{k}\right), s\right) N\left(x_{k}, t\right)\right] x_{k}\right), t\right) \geq \frac{t}{t+\psi\left(x_{1}, x_{1}, \ldots, x_{k}, x_{k}\right)},
\end{aligned}
$$

then $(E, N)$ is a fuzzy $C^{*}$-algebra with involution $x^{*}=H(x)$ for all $x \in E$.

Proof. Let $(S, d)$ be the complete generalized metric space defined in the proof of Theorem 2 . Consider the linear mapping $J: S \rightarrow S$ by

$$
J g(x):=2 g\left(\frac{x}{2}\right) \quad \forall x \in E .
$$

Putting $\lambda=1$ and $y_{1}=\ldots=y_{k}=0$ in (12), we have

$$
N_{k}\left(\left(2 f\left(\frac{x_{1}}{2}\right)-f\left(x_{1}\right), \ldots, 2 f\left(\frac{x}{k}\right)-f\left(x_{k}\right)\right), t\right) \geq \frac{t}{t+\psi\left(x_{1}, 0, \ldots, x_{k}, 0\right)},
$$


for all $x_{1}, . . x_{k} \in E$ and all $t>0$. Therefore $d(J f, f) \leq 1$ and thus

$$
d(f, H) \leq \frac{1}{1-L^{\prime}}
$$

which implies that the inequality (15) holds. The rest of the proof is similar to the proof of Theorem 2.

Corollary 1. Let $(E, N)$ be a fuzzy Banach algebra and $\left\{\left(E^{K}, N_{k}\right), k \in \mathbb{N}\right\}$ be a multi-fuzzy Banach algebra. In addition, let $p \in(0,1)$ and $\theta \in[0, \infty)$ be real numbers. Suppose that $f: E \rightarrow E$ with $f(1)=1$, satisfies satisfying

$$
\begin{gathered}
N_{k}\left(\left(D_{\lambda \gamma} f\left(x_{1}, y_{1}\right), D_{\lambda \gamma} f\left(x_{2}, y_{2}\right), \ldots, D_{\lambda \gamma} f\left(x_{k}, y_{k}\right)\right), t\right) \geq \frac{t}{t+\theta \sum_{i=1}^{k}\left(\left\|x_{i}\right\|^{p}+\left\|y_{i}\right\|^{p}\right)^{p}} \\
N_{k}\left(\left(f\left(x_{1} y_{1}\right)-f\left(y_{1}\right) f\left(x_{1}\right), \ldots, f\left(x_{k} y_{k}\right)-f\left(y_{k}\right) f\left(x_{k}\right)\right), t\right) \geq \frac{t}{t+\theta \sum_{i=1}^{k}\left(\left\|x_{i}\right\|^{p}+\left\|y_{i}\right\|^{p}\right)^{\prime}}, \\
N-\lim _{k \rightarrow \infty} 2^{-k} f\left(2^{k}\left(N-\lim _{k \rightarrow \infty} 2^{-k} f\left(2^{k} x\right)\right)\right)=x,
\end{gathered}
$$

for all $x_{1}, \ldots, x_{k}, y_{1}, \ldots, y_{k} \in E$, all $t>0$ and all $\lambda, \gamma \in \mathbb{T}_{\frac{1}{m_{0}}}^{1}$. Then there exists a unique involution $H: E \rightarrow$ E such that

$$
H(x):=N-\lim _{k \rightarrow \infty} \frac{1}{2^{k}} f\left(2^{k} x\right)
$$

and

$$
N_{k}\left(\left(f\left(x_{1}\right)-H\left(x_{1}\right), \ldots, f\left(x_{k}\right)-H\left(x_{k}\right)\right), t\right) \geq \frac{\left(1-2^{p-1}\right) t}{\left(1-2^{p-1}\right) t+2^{p-1} \theta \sum_{i=1}^{k}\left\|x_{i}\right\|^{p}} .
$$

Further, if

$$
N_{k}\left(\left(\left[N\left(f\left(x_{1}\right), t\right)-N\left(x_{1}, t\right)\right] x_{1}, \ldots,\left[N\left(f\left(x_{k}\right), t\right)-N\left(x_{k}, t\right)\right] x_{k}\right), t\right) \geq \frac{t}{t+2 \theta \sum_{i=1}^{k}\left\|x_{i}\right\|^{p}},
$$

for all $x_{1}, \ldots, x_{k} \in E$ and for all $t>0$, then $(E, N)$ is a fuzzy Banach $*$-algebra.

Moreover, if

$$
\begin{aligned}
& N_{k}\left(\left(\left[N\left(f\left(x_{1}\right) x_{1}, s t\right)-N\left(f\left(x_{1}\right), s\right) N\left(x_{1}, t\right)\right] x_{1}, \ldots,\right.\right. \\
& \left.\left.\left[N\left(f\left(x_{k}\right) x_{k}, s t\right)-N\left(f\left(x_{k}\right), s\right) N\left(x_{k}, t\right)\right] x_{k}\right), t\right) \geq \frac{t}{t+2 \theta \sum_{i=1}^{k}\left\|x_{i}\right\|^{p}},
\end{aligned}
$$

for all $x_{1}, \ldots, x_{k} \in E$ and for all $s, t>0$, then $(E, N)$ is a fuzzy $C^{*}$-algebra with involution $x^{*}=H(x)$ for all $x \in E$.

Proof. It follows from Theorem 2 by putting

$$
\left.\psi\left(x_{1}, y_{1}, \ldots, x_{k}, y_{k}\right)=\theta \sum_{i=1}^{k}\left\|x_{i}\right\|^{p}+\left\|y_{i}\right\|^{p}\right),
$$

for all $x_{1}, \ldots, x_{k}, y_{1}, \ldots, y_{k} \in E$ and $L=2^{p-1}$. 
Corollary 2. Let $(E, N)$ be a fuzzy Banach algebra and $\left\{\left(E^{K}, N_{k}\right), k \in \mathbb{N}\right\}$ be a multi-fuzzy Banach algebra. In addition, let $p \in(0,1)$ and $\theta \in[0, \infty)$ be real numbers. Suppose that $f: E \rightarrow E$ with $f(1)=1$, satisfies

$$
\begin{gathered}
N_{k}\left(\left(D_{\lambda \gamma} f\left(x_{1}, y_{1}\right), D_{\lambda \gamma} f\left(x_{2}, y_{2}\right), \ldots, D_{\lambda \gamma} f\left(x_{k}, y_{k}\right)\right), t\right) \geq \frac{t}{t+\theta \sum_{i=1}^{k} \sum_{j=1}^{k}\left\|x_{i} y_{j}\right\|^{p^{p}}}, \\
N_{k}\left(\left(f\left(x_{1} y_{1}\right)-f\left(y_{1}\right) f\left(x_{1}\right), \ldots, f\left(x_{k} y_{k}\right)-f\left(y_{k}\right) f\left(x_{k}\right)\right), t\right) \geq \frac{t}{t+\theta \sum_{i=1}^{k} \sum_{j=1}^{k}\left\|x_{i} y_{j}\right\|^{p}}, \\
N-\lim _{k \rightarrow \infty} 2^{-k} f\left(2^{k}\left(N-\lim _{k \rightarrow \infty} 2^{-k} f\left(2^{k} x\right)\right)\right)=x,
\end{gathered}
$$

for all $x_{1}, \ldots, x_{k}, y_{1}, \ldots, y_{k} \in E$, all $t>0$ and all $\lambda, \gamma \in \mathbb{T}_{\frac{1}{m_{0}}}^{1}$. Then $f$ is an involution on $E$. Moreover, if

$$
N_{k}\left(\left(\left[N\left(f\left(x_{1}\right), t\right)-N\left(x_{1}, t\right)\right] x_{1}, \ldots,\left[N\left(f\left(x_{k}\right), t\right)-N\left(x_{k}, t\right)\right] x_{k}\right), t\right) \geq \frac{t}{t+\theta\left(\sum_{i=1}^{k}\left\|x_{i}\right\|^{p}\right)^{2}},
$$

for all $x_{1}, \ldots, x_{k} \in E$ and for all $t>0$, then $(E, N)$ is a fuzzy Banach $*$-algebra.

Furthermore, if

$$
\begin{aligned}
& N_{k}\left(\left(\left[N\left(f\left(x_{1}\right) x_{1}, s t\right)-N\left(f\left(x_{1}\right), s\right) N\left(x_{1}, t\right)\right] x_{1}, \ldots,\right.\right. \\
& \left.\left.\left[N\left(f\left(x_{k}\right) x_{k}, s t\right)-N\left(f\left(x_{k}\right), s\right) N\left(x_{k}, t\right)\right] x_{k}\right), t\right) \geq \frac{t}{t+\theta\left(\sum_{i=1}^{k}\left\|x_{i}\right\|^{p}\right)^{2}},
\end{aligned}
$$

for all $x_{1}, \ldots, x_{k} \in E$ and for all $s, t>0$, then $(E, N)$ is a fuzzy $C^{*}$-algebra with involution $x^{*}=H(x)$ for all $x \in E$.

Proof. We put

$$
\psi\left(x_{1}, y_{1}, \ldots, x_{k}, y_{k}\right):=\theta \sum_{i=1}^{k} \sum_{j=1}^{k}\left\|x_{i} y_{j}\right\|^{p}
$$

for all $x_{1}, \ldots, x_{k}, y_{1}, \ldots, y_{k} \in E$ and $L=2^{2 p-1}$ in Theorem 2, and then, as a result, the sentence is obtained.

\section{Conclusions}

We define multi-fuzzy Banach algebra and then prove the Hyers-Ulam-Rassias stability of involution on multi-fuzzy Banach algebra by fixed point method and find some conditions for which a multi-Banach algebra with approximate involution is a $C^{*}$-algebra.

Author Contributions: Conceptualization, E.M.; methodology, P.L.; validation, P.L., E.M. and M.D.l.S.; investigation, P.L. and E.M.; writing-original draft preparation, P.L. and E.M.; writingreview and editing, M.D.1.S.; supervision, E.M.; project administration, M.D.1.S.; funding acquisition, M.D.l.S. All authors have read and agreed to the published version of the manuscript.

Funding: This research was supported by Basque Government, Grant IT1207-19.

Institutional Review Board Statement: Not applicable.

Informed Consent Statement: Not applicable.

Data Availability Statement: Not applicable.

Conflicts of Interest: The authors declare no conflict of interest. 


\section{References}

1. Dales, H.G.; Moslehian, M.S. Stability of mapping on multi-normed spaces. Glasg. Math. J. 2007, 49, 321-332. [CrossRef]

2. Moslehian, M.S. Superstability of higher derivations in multi-Banach algebras. Tamsui Oxf. J. Math. Sci. 2008, 24, 417-427.

3. Moslehian, M.S.; Nikodem, K.; Popa, D. Asymptotic aspect of the quadratic functional equation inmulti-normed spaces. J. Math. Anal. Appl. 2009, 355, 717-724. [CrossRef]

4. Dales, H.G.; Polyakov, M.E. Multi Normed Spaces and Multi-Banach Algebras; University of Leeds: Leeds, UK, 2008. Available online: https: / / www1.maths.leeds.ac.uk/pure/analysis/conf1/dales.pdf (accessed on 20 November 2021).

5. Ulam, S.M. A Collection of Mathematical Problems; Interscience: New York, NY, USA, 1960.

6. Hyers, H.D. On the stability of the linear functional equation. Proc. Natl. Acad. Sci. USA 1941, 27, 222-224. [CrossRef] [PubMed]

7. Rassias, T.M. On the stability of the linear mapping in Banach spaces. Proc. Natl. Acad. Sci. USA 1978, 72, 297-300. [CrossRef]

8. Jung, S.M. Hyers-Ulam-Rassias stability of Jensen's equation and its application. Proc. Am. Math. Soc. 1998, 126, 3137-3143. [CrossRef]

9. Movahednia, E. Fuzzy Stability of Quadratic Functional Equations in General Cases ISRN Math. Anal. Hindawi 2011, 10, 553-560. [CrossRef]

10. Mosadegh, S.M.S.M.; Movahednia, E. Stability of preserving lattice cubic functional equation in Menger probabilistic normed Riesz spaces. J. Fixed Point Theory Appl. 2018, 20, 34-43. [CrossRef]

11. Movahednia, E.; Mursaleen, M. Stability of a generalized quadratic functional equation in intuitionistic fuzzy 2-normed space. Filomat 2016, 30, 449-457. [CrossRef]

12. Movahednia, E.; Mosadegh, S.M.S.M.; Park, C.; Shin, D. Stability of a lattice preserving functional equation on Riesz space: Fixed point alternative. J. Comput. Anal. Appl. 2016, 21, 83-89.

13. Mirzavaziri, M.; Moslehian, M.S. A fixed point approach to stability of a quadratic equation. Bull. Braz. Math. Soc. 2006, 37, 361-376. [CrossRef]

14. Radu, V. The fixed point alternative and the stability of functional equations. Fixed Point Theory 2003, 4, 91-96.

15. Cadariu, L.; Radu, V. Fixed points and the stability of Jensen's functional equation. J. Inequal. Pure Appl. Math. 2003, 4, 4.

16. Diaz, J.; Margolis, B. A fixed point theorem of the alternative for contractions on a generalized complete metrice space. Bull. Am. Math. Soc. 1968, 74, 305-309. [CrossRef]

17. Bag, T.; Samanta, S.K. Finite dimensional Fuzzy normed linear spaces. J. Fuzzy Math. 2003, 11, 687-705.

18. Park, C.; Gorgji, M.E.; Saadati, R. Random homomorphisms and random derivations in random normed algebras via fixed point method. J. Inequal. Appl. 2012, 2012, 194. [CrossRef]

19. Khanehgir, M. Stability of the Jenesen's functional equation in multi-fuzzy normed space. Iran. J. Fuzzy Syst. 2017, 14, 105-119.

20. $\mathrm{Lo}^{\prime} \mathrm{lo}^{\prime}$, P.; Movahednia, E.; De la sen, M. Approximation of involution in new fuzzy $C^{*}$-Algebras: fixed point technique. Fixed Point Theory 2022, accepted.

21. Mihet, D.; Radu, V. On the stability of the additive Cauchy functional equation in random normed spaces. J. Math. Anal. Appl. 2008, 343, 567-572. [CrossRef]

22. Gordji, M.E. Nearly involutions on Banach algebras; A fixed point approach. Fixed Point Theory 2013, 14, 117-124. 